\title{
Renal malignant tumor with the loss of INI1 expression and WT1 positive in a 3-year-old boy: a case report
}

\author{
Hengyou Wang ${ }^{1}$, Daxing Tang ${ }^{1}$, Dehua $\mathrm{Wu}^{1}$, Chang Tao ${ }^{1}$, Guangjie Chen ${ }^{1}$, Wei Ru ${ }^{1}$, Kun Zhu ${ }^{2}$ \\ ${ }^{1}$ Department of Urology, the Children's Hospital, Zhejiang University School of Medicine, National Clinical Research Center for Child Health, \\ Hangzhou, China; ${ }^{2}$ Department of Pathology, the Children's Hospital, Zhejiang University School of Medicine, National Clinical Research Center \\ for Child Health, Hangzhou, China \\ Correspondence to: Daxing Tang. Department of Urology, the Children's Hospital, Zhejiang University School of Medicine, National Clinical Research \\ Center For Child Health, 57 Zhugan lane, Hangzhou 310003, China. Email: tangdx0206@zju.edu.cn.
}

\begin{abstract}
Wilms' tumor is the most common primary renal malignancy in children (80\%) and the less common tumors include renal cell carcinoma, rhabdoid tumor, clear cell sarcoma, cellular congenital mesoblastic nephroma and medullary carcinoma, all of which originate from renal parenchyma. The tumors originating from renal pelvis are rare. The immunohistochemistry (IHC) showed INI1 deletion with the WT1 positive which has not been reported as we know. A 3-year-old boy was admitted to hospital for vomiting. An ultrasonography examination revealed a mass in the right kidney, medium echo, as well as hydronephrosis with collecting system separation of $3.5 \mathrm{~cm}$. The computed tomography and the magnetic resonance $(M R)$ radical showed that the tumor occupied the right renal pelvis and extended into the ureter. A radical nephroureterectomy was accomplished through a transabdominal approach. The pathologic diagnosis was malignant renal tumor with INI1 deficiency which was atypical in morphology and immunophenotype, but according to immunophenotype renal rhabdomyoid tumor could not be excluded. The patient was treated with carboplatin, etoposide and cyclophosphamide chemotherapy for 6 months. Follow-up studies of the patient showed no indication of recurrence or metastasis 22 months after nephrectomy. The novel findings may expand the spectrum of pediatric renal tumors to include the special malignancy.
\end{abstract}

Keywords: Integrase interactor 1 (INI1); Wilms' tumor 1 (WT1); renal malignant tumor; children; case report

Submitted Apr 01, 2020. Accepted for publication Sep 10, 2020.

doi: $10.21037 /$ tau-20-834

View this article at: http://dx.doi.org/10.21037/tau-20-834

\section{Introduction}

Wilms' tumor is the most common primary renal malignancy in children $(80 \%)$; the less common tumors include renal cell carcinoma, rhabdoid tumor, clear cell sarcoma, cellular congenital mesoblastic nephroma and medullary carcinoma, each of which account for approximately $2 \%$ to $5 \%$ of pediatric renal tumors (1). The above two groups of tumors usually originate from the renal parenchyma and can invade the renal sinus sometimes. The tumor was located in the renal pelvis in this case and the immunohistochemistry (IHC) showed that integrase interactor 1 (INI1) deletion with the Wilms' tumor 1 (WT1) positive which has not been reported as we know.
The prototypical INI-1-deficient tumor is the malignant rhabdoid tumor (MRT) which was first described in the kidney of infancy but also occurs in soft tissue, viscera, and the brain $(2,3)$. In addition to MRT, pediatric tumors with complete loss of INI1 expression include atypical teratoid rhabdoid tumor, renal medullary carcinoma, myoepithelial carcinoma, cribriform neuroepithelial tumor (4-7). Tumors occurring in children's kidney are MRT and medullary carcinoma of which $W T 1$ is negative. WT1 gene was first identified as a strong candidate predisposition gene for Wilms' tumor which retains INI1 expression $(2,8)$. We report a case of primary renal malignant tumor with INI1 deletion and $W T 1$ positive in IHC.

We present the following article in accordance with 

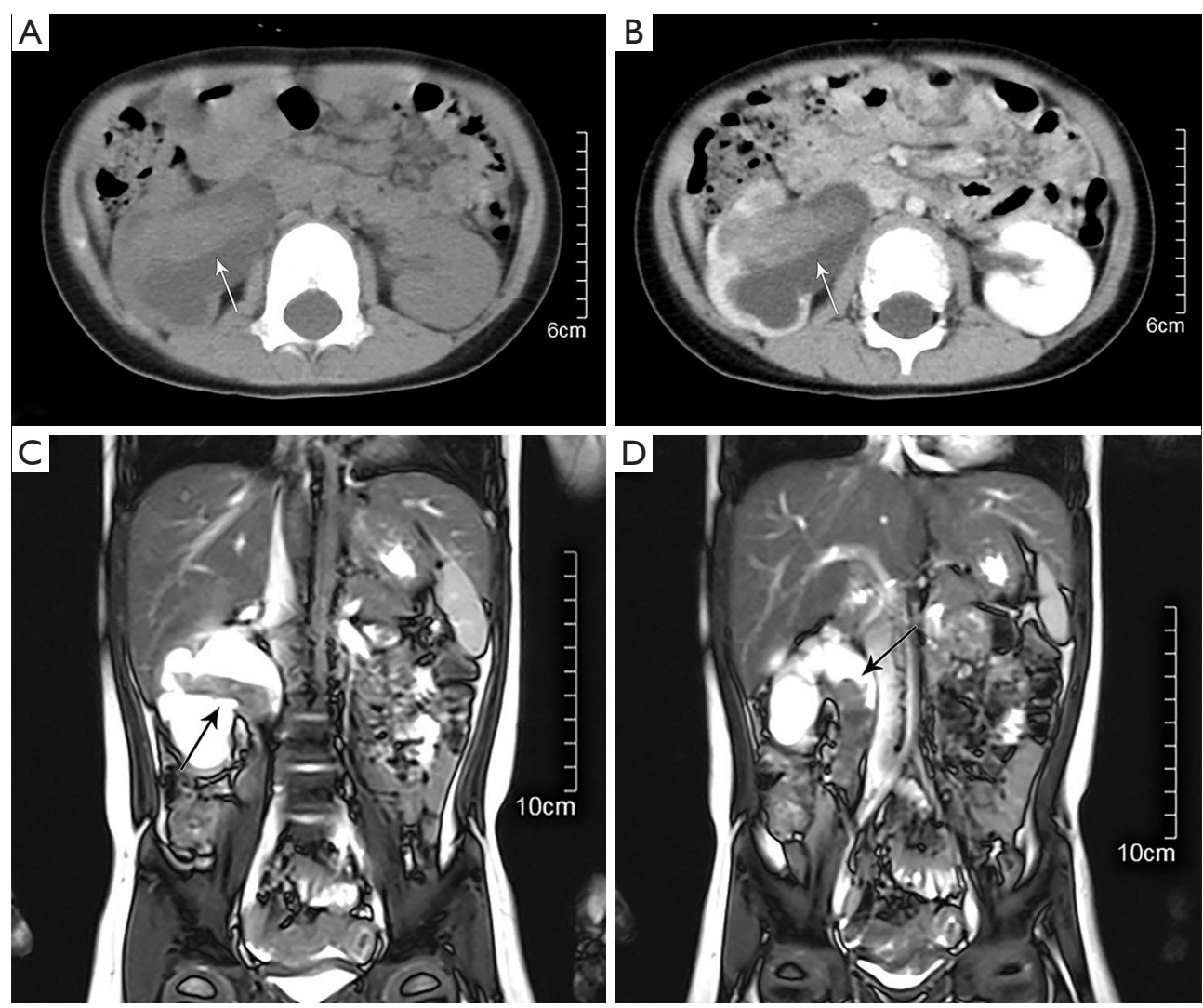

Figure 1 Preoperative CT and MR. (A) No-contrasted CT scan illustrated enlarged inhomogeneous high-density mass, occupying the right renal pelvicalyceal system. (B) Contrasted-enhanced CT showed that the mass was enhanced slightly. Coronal MR showed the tumor originated from the renal pelvis (C) and extended into the proximal ureter (D).

the CARE reporting checklist (available at http://dx.doi. org/10.21037/tau-20-834).

\section{Case presentation}

A 3-year-old boy was admitted to hospital for vomiting without abdominal pain or gross hematuria and with no definite signs for physical examination. The patient denied any significant past medical history and the family history of tumor. An ultrasonography examination revealed a mass, measuring $3.5 \times 2.8 \times 1.6 \mathrm{~cm}$ in the right kidney, medium echo, as well as hydronephrosis with collecting system separation of $3.5 \mathrm{~cm}$. Further abdominal enhanced computed tomography (CT) showed a mixed density mass in the right pelvis and upper ureter with mild enhancement which was lower than renal parenchyma (Figure 1A,B). Lymph nodes enlargement were found in the renal hilus. The magnetic resonance (MR) clearly showed that the renal pelvis tumor extended into the proximal ureter on coronal sectional image (Figure 1C,D). No distant metastasis was found by chest CT and bone X-ray of extremities. Urinalysis results showed 2 red blood cells and 12 white blood cells per high power field. A diagnosis of right renal pelvis tumor, stage III, was considered after multidisciplinary discussion.

A radical nephroureterectomy was accomplished through a transabdominal approach. During the operation, a mass measuring $9 \times 2 \times 1.5 \mathrm{~cm}$ was found in the pelvis from calyx growing into the upper ureter which shaped like a cone with no capsule (Figure $2 A$ ). The proximal segment looked like fleshy texture and the distal part was seen yellowish coverage on the surface (Figure 2B). The renal hilar lymph nodes, renal artery and vein were free from tumor. Meanwhile, an ectopic appendix was found in the right upper abdomen.

Histologically (Figure 3), the tumor was composed of round, polygonal or irregular tumor cells with obvious mucoid matrix. The nuclei were round-to-oval with inconspicuous nucleoli. Patchy mitoses and massive necrosis 

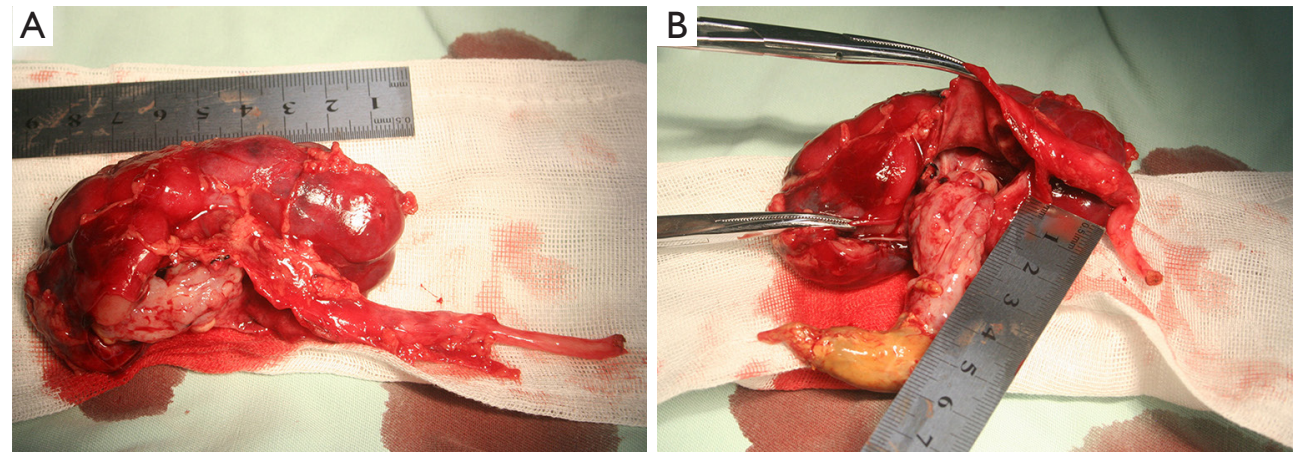

Figure 2 Gross appearance of the tumor. (A) The tumor occupied the right renal pelvicaliceal system and ureter. (B) The tumor was red and gray with fleshy texture shaped like a cone.
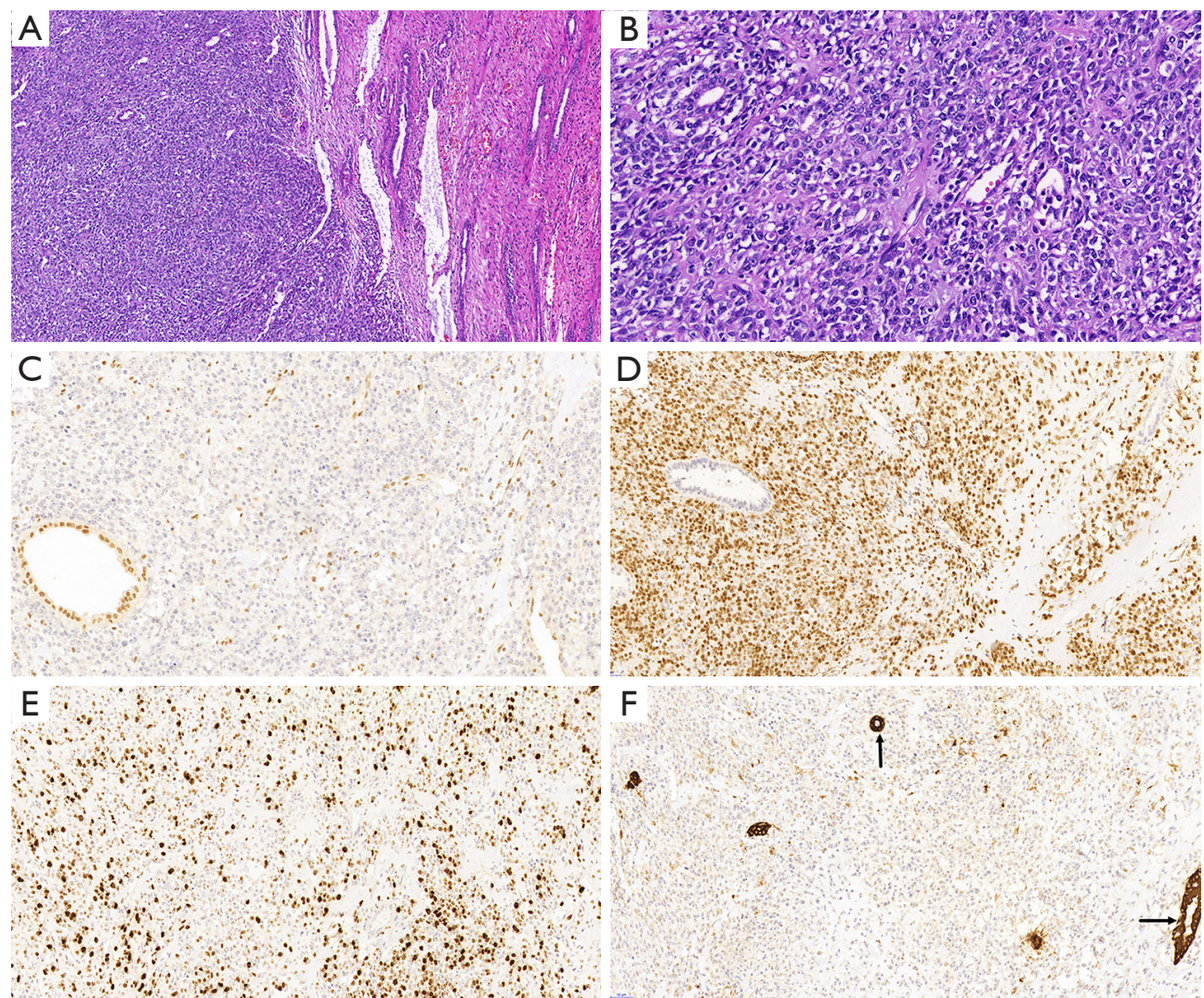

Figure $3 \mathrm{H} \& \mathrm{E}$ stained (A: 100×; B: 400×). (A) Well-defined pushing margin was caused by a continuous front of tumor cells. (B) Round, polygonal or irregular tumor cells showed and round-to-oval nuclei with inconspicuous nucleoli. IHC stained (200×). (C) INI1 protein expression was lost in the tumor cells. (D) WT1 showed diffuse expression in nucleus of the tumor cells. (E) More than $45 \%$ of the tumor cells were positive for Ki-67. (F) Scattered tubules were positive for CK, suggesting the origin from renal tubule epithelium. 


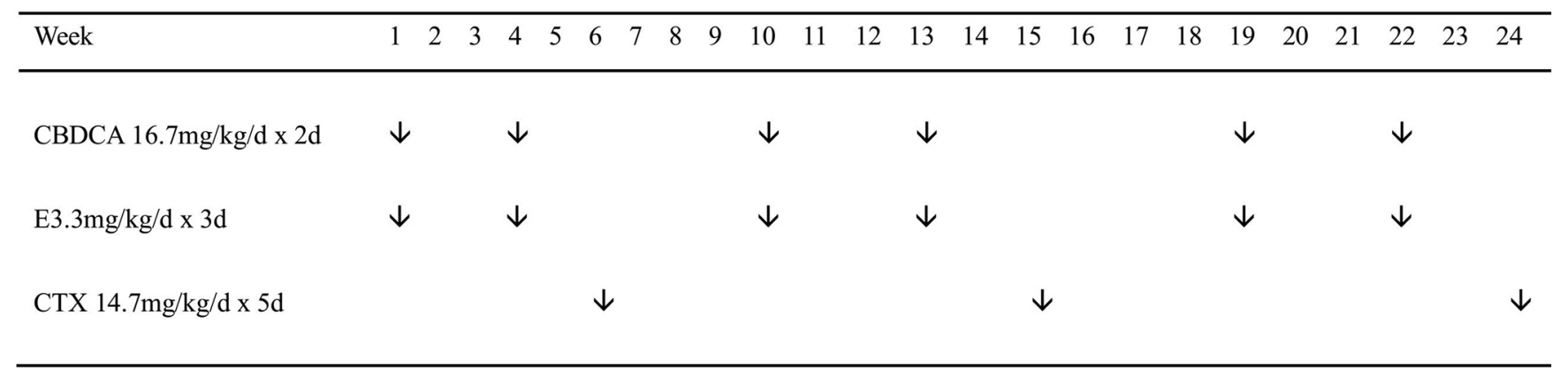

Figure 4 Chemotherapy protocol for patient. CBDCA, carboplatin; E, etoposide; CTX, cyclophosphamide.

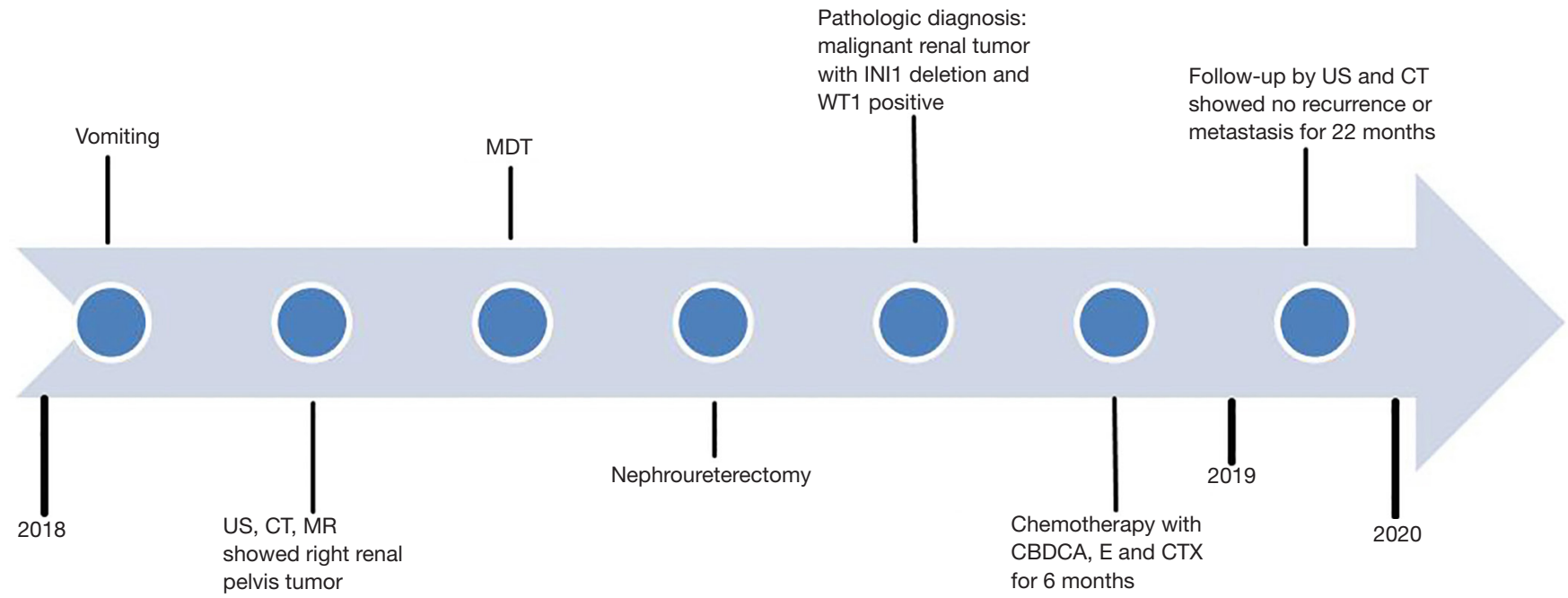

Figure 5 The timeline picture of historical and current information. US, ultrasonography; MDT, multidisciplinary team.

were present. Meanwhile, focal areas with calcification were noted. IHC stains revealed expression of $W T 1$, cytokeratin(CK), S-100, Vimentin, CD99, CD34, Ki$67(45 \%)$, Calponin, smooth muscle actin (SMA), and no expression of INI1, HMB45, epithelial membrane antigen (EMA), CD10, ALK, glial fibrillary acidic protein (GFAP), Cyclin-D1 (Figure 3). The pathologic diagnosis was right renal malignant tumor which rhabdoid tumor or myoepithelial carcinoma was first considered with INI1 deletion in our hospital. For further diagnosis pathological slides were sent to Shanghai Tumor Hospital which is famous in China. The final result was malignant renal tumor with INI1 deficiency which was atypical in morphology and immunophenotype, but according to immunophenotype renal rhabdomyoid tumor could not be excluded. In addition, no clinically significant mutation of oncogene was found in tumor gene detection.

The patient was treated with carboplatin, etoposide and cyclophosphamide chemotherapy according to the regimen of the National Wilms' Tumor Study Group 5 (Figure 4) without obvious adverse drug reactions except for occasional vomiting and mild myelosuppression. The child was in good health and developed normally by the scales of children development. The 22-month follow-up included ultrasonography examinations at 1, 2, 3, 6, 9, 12, 15, 21 months and CT scan at $6,10,15,21$ months postoperatively (Figure 5). So far, no local recurrence or metastasis has been observed.

All procedures performed in studies involving human participants were in accordance with the ethical standards of the institutional and/or national research committee(s) and with the Helsinki Declaration (as revised in 2013). Written 
informed consent was obtained from the patient.

\section{Discussion}

INI1 has been known by a variety of names, including SMARCB1, hSNF5 and BAF47 (BRM or BRG1 associated factors subunit of $47 \mathrm{kDa})(2,4,9)$. INI1 is an important tumor suppressor gene which is a core subunit of the SWI/ SNF ATP-dependent chromatin remodeling complex encoded at chromosomal position $22 \mathrm{q} 11.2(2,10)$. It is expressed in the nuclei of all normal cells and can be readily identified using IHC (11). Loss of expression of the protein permitted the development of an IHC assay that is based on the loss of nuclear expression of the protein in tumor cells $(12,13)$. Patients with germline mutations or deletions affecting INI1 are predisposed to the development of rhabdoid tumors (12).

What does it mean when a newly diagnosed patient with loss of expression of INI1? Margol gave the opinion that: in patients under the age of 3 years with tumors occurring in kidney, it is most likely that this is an rhabdoid tumor that will be biologically aggressive; while tumors occurring in patients greater than 3 years of age with nonrenal locations and without typical rhabdoid features are unlikely to be rhabdoid tumor and therefore may have less aggressive phenotypes (14). In this case, the renal tumor occurred in a 3-year-old boy with loss of INI1, so rhabdoid tumor was first considered in this case.

Rhabdoid tumor of the kidney (RTK) is a rare but highly lethal malignant tumor that accounts for less than $2 \%$ of kidney tumors in infants and young children (15). Cytologically, a key feature is the "rhabdoid" cell, which is characterized by large and ovoid-to-polygonal, with an eccentrically placed vesicular nucleus which contains a very large inclusion-like nucleolus. The cytoplasm tends to be abundant and eosinophilic, with a perinuclear pale, spherical inclusion corresponding to aggregates of intermediate filaments $(2,4,14)$. The number of such classic "rhabdoid" cells can vary considerably within a given tumor and can vary tremendously from tumor to tumor (3). Some rhabdoid tumors are composed almost completely of these cells, some have very few, and some may have none, at all. Mitotic activity is usually very high, and atypical mitoses may be numerous (4). Because of this, lack of classic "rhabdoid" cells within an undifferentiated tumor does not exclude the possibility of rhabdoid tumor.

By IHC, nearly all express vimentin and the majority express EMA and/or broad spectrum cytokeratins (CK).
There may be variable expression of synaptophysin, GFAP, SMA, desmin, CD34 and CD99. Loss of INI expression is a constant finding in RTK $(2,4)$. In this case the positive indicator including vimentin, CK, SMA, CD34, CD99, as well as INI1 negative, which similar to RTK. However, the positive expression of $W T 1$ is different from MRT.

Another diagnosis is myoepithelial carcinoma which is considered. Usually salivary gland pleomorphic adenoma is the best-recognized myoepithelial tumor with the loss of INI1 expression (2). Cajaiba first reported two examples of primary renal myoepithelial carcinoma in 2016, both occurring in children (1). Histologically, a nonencapsulated, infiltrative neoplasm demonstrated heterogenous cytologic features varying from larger, round to polygonal cells with coarse chromatin, prominent nucleoli, and eosinophilic cytoplasm to smaller cells with round to oval nuclei, mild cytologic atypia, and scant to abundant clear cytoplasm. These cells were embedded in a myxoid stroma and foci of necrosis and many mitotic figures were appreciated. Immunohistochemical stains performed on the nephrectomy specimen revealed expression of pan-cytokeratin, S-100, vimentin, patchy expression CD10, and no expression of WT1, CD56, calponin, SMA and NGFR. INI1 stain showed nuclear retention in tumor cells.

In this case IHC showed that S-100, vimentin and CK were all positive, similar to myoepithelial carcinoma, but INI1 was negative with expression of WT1. Also, there was no typical cytological feature in histology. The tumor originated from pelvis while myoepithelial carcinoma occurred in renal parenchyma, so we don't think the diagnosis of myoepithelial carcinoma is inappropriate according to the above characteristics.

To our knowledge, the renal pelvis tumor with INI1 deletion and WT1 positive has not been reported. The spectrum of histopathologic and immunophenotypic changes seen in this case did not fit into any known entity within pediatric renal tumors. As the exophytic growth of tumor into the pelvis caused hydronephrosis, the case presented with early stage and no distant metastases at the time of diagnosis. Thus, the prognosis was good after treatment with radical nephroureterectomy and chemotherapy. Unfortunately, no significant mutation of oncogene was found. The follow-up time is limited and further observation is needed.

In summary, loss of INI1 expression in kidney is sensitive, but it is not specific. Correlation with histologic and immunophenotypic findings, the patient's age, and the location of the tumor should be required to make a 
clinical diagnosis. The tumor was located in the renal pelvis with the INI1 deletion and $W T 1$ positive, which may be recognized as part of the spectrum of pediatric renal tumors.

\section{Acknowledgments}

Funding: This work was supported by the National Key Research and Development Project [grant numbers: 2018 YFC1002702].

\section{Footnote}

Reporting Checklist: The authors have completed the CARE reporting checklist. Available at http://dx.doi.org/10.21037/ tau-20-834

Conflicts of Interest: All authors have completed the ICMJE uniform disclosure form (available at http://dx.doi. org/10.21037/tau-20-834). The authors have no conflicts of interest to declare.

Ethical Statement: The authors are accountable for all aspects of the work in ensuring that questions related to the accuracy or integrity of any part of the work are appropriately investigated and resolved. All procedures performed in studies involving human participants were in accordance with the ethical standards of the institutional and/or national research committee(s) and with the Helsinki Declaration (as revised in 2013). Written informed consent was obtained from the patient.

Open Access Statement: This is an Open Access article distributed in accordance with the Creative Commons Attribution-NonCommercial-NoDerivs 4.0 International License (CC BY-NC-ND 4.0), which permits the noncommercial replication and distribution of the article with the strict proviso that no changes or edits are made and the original work is properly cited (including links to both the formal publication through the relevant DOI and the license). See: https://creativecommons.org/licenses/by-nc-nd/4.0/.

\section{References}

1. Cajaiba MM, Jennings LJ, Rohan SM, et al. Expanding the Spectrum of Renal Tumors in Children: Primary Renal Myoepithelial Carcinomas With a Novel EWSR1-KLF15 Fusion. Am J Surg Pathol 2016;40:386-94.
2. Hollmann TJ, Hornick JL. INI1-deficient tumors: diagnostic features and molecular genetics. Am J Surg Pathol 2011;35:e47-63.

3. White FV, Dehner LP, Belchis DA, et al. Congenital disseminated malignant rhabdoid tumor: a distinct clinicopathologic entity demonstrating abnormalities of chromosome 22q11. Am J Surg Pathol 1999;23:249-56.

4. Pawel BR. SMARCB1-deficient Tumors of Childhood: A Practical Guide. Pediatr Dev Pathol 2018;21:6-28.

5. Parham DM, Weeks DA, Beckwith JB. The clinicopathologic spectrum of putative extrarenal rhabdoid tumors. An analysis of 42 cases studied with immunohistochemistry or electron microscopy. Am J Surg Pathol 1994;18:1010-29.

6. Wick MR, Ritter JH, Dehner LP. Malignant rhabdoid tumors: a clinicopathologic review and conceptual discussion. Semin Diagn Pathol 1995;12:233-48.

7. Gleason BC, Fletcher CD. Myoepithelial carcinoma of soft tissue in children: an aggressive neoplasm analyzed in a series of 29 cases. Am J Surg Pathol 2007;31:1813-24.

8. Hastie ND. Wilms' tumour 1 (WT1) in development, homeostasis and disease. Development 2017;144:2862-72.

9. Kalimuthu SN, Chetty R. Gene of the month: SMARCB1. J Clin Pathol 2016;69:484-9.

10. Roberts CW, Orkin SH. The SWI/SNF complex-chromatin and cancer. Nat Rev Cancer 2004;4:133-42.

11. Hoot AC, Russo P, Judkins AR, et al. Immunohistochemical analysis of hSNF5/INI1 distinguishes renal and extra-renal malignant rhabdoid tumors from other pediatric soft tissue tumors. Am J Surg Pathol 2004;28:1485-91.

12. Geller JI, Roth JJ, Biegel JA. Biology and Treatment of Rhabdoid Tumor. Crit Rev Oncog 2015;20:199-216.

13. Agaimy A. The expanding family of SMARCB1(INI1)deficient neoplasia: implications of phenotypic, biological, and molecular heterogeneity. Adv Anat Pathol 2014;21:394-410.

14. Margol AS, Judkins AR. Pathology and diagnosis of SMARCB1-deficient tumors. Cancer Genetics 2014;207:358-64.

15. Chang CJ, Yeh ML, Chen CC. Rhabdoid tumor of the kidney with spontaneous rupture: case report and review of literature. Pediatr Surg Int 2008;24:451-3.

Cite this article as: Wang $\mathrm{H}$, Tang $\mathrm{D}, \mathrm{Wu} \mathrm{D}$, Tao C, Chen $\mathrm{G}, \mathrm{Ru}$ W, Zhu K. Renal malignant tumor with the loss of INI1 expression and WT1 positive in a 3-year-old boy: a case report. Transl Androl Urol 2020;9(5):2275-2280. doi:10.21037/tau-20-834 